\title{
Regards sur l'Orthopédie-Traumatologie
}

\author{
G. Piganiol
}

Centre de Rééducation "Les Rosiers", 45, boulevard Henri Bazin, F-21000 Dijon

Lorsqu'après plus d'un demi siècle de vie médicale, on jette un regard rétrospectif sur ce que fut l'évolution de notre profession, on ne peut échapper à un certain vertige.

Au début de nos études, la thérapeutique se résumait à à peine plus d'une dizaine de drogues efficaces, et encore !

Les sulfamides rouges "Prontosil" et "Rubiazol" étaient juste découvertes, le "Dagenan" voyait le jour, et nos Maîtres étaient éblouis par les progrès de la lutte contre l'infection.

Les bienfaits médicaux de la colonisation maîtrisaient les grandes épidémies d'Afrique et d'Extrême Orient, variole, peste, fièvre jaune, maladie du sommeil.

Dans notre spécialité de Chirurgie de l'appareil loco-moteur, l'ostéosynthèse balbutiait. Parham, Shermann, les frères Lambotte puis Danis avaient défriché un terrain qui se montrerait fertile, et je n'oserai dire jusqu'à quelle date j'ai acheté une partie de mon instrumentation fabriquée par Peugeot chez le quincailler de l'angle de la rue.

Puis ce fut la perfection de l'A.O., basée sur des idées lumineuses et simples, et nous nous sentions alors presque ridicules de ne pas les avoir eues nous mêmes.

Les voyages à Montbéliard puis les pélerinages à Davos faisaient de nous des adeptes, voir même des prosélytes de "La méthode" que d'autres rejetaient parcequ'ils ne l'avaient pas inventée.

L'enclouage centro-médullaire nous était arrivé d'Allemagne par des prisonniers porteurs de clous qui séduisaient les audacieux, mais révoltaient ceux qui imaginaient on ne sait quelle expérimentation in anima vili.

Mais accompagnant les progrès techniques, les diverses spécialités médicales voyaient leurs frontières évoluer.

A la grande époque de la scoliose, des maux de Pott, des tumeurs blanches, des luxations congénitales et du rachitisme, l'Orthopédie était fille de 1a Chirurgie Infantile dont elle continuait l'oeuvre dans le temps chez les adultes.

Au Pavillon T de Grange Blanche, Monsieur Tavernier avait comme Assistants P. Truchet et A. Trillat, comme Interne G. Demourgues. J'y étais Externe ou "Hypo" et accroupi sous les champs, manoeuvrant l'Ombredanne, j'écoutais avec ravissement des mots que j'essayais de comprendre et que mes Anciens m'apprenaient avec une patience et une amitié sans faille.

Les progrès de la pédiatrie et de la prophylaxie des malformations, la diminution énorme des infections tuberculeuses ostéo-articulaires semblaient devoir stériliser une spécialité qui paraissait vouée au déclin.

C'est alors que nos leaders imaginaient de s'amputer de la Chirurgie Infantile pour lancer une vaste OPA et créer "L'Orthopédie Traumatologie, Chirurgie Réparatrice et Reconstructive". Ils pensaient ainsi sauver une spécialité moribonde, au moment au contraire où celle-ci allait exploser dans la voie des prothèses, tracée en France par mon cher ami Robert Judet et son frère.

Les jeunes Chirurgiens Orthopédiques débarquaient en force dans nos Cliniques et nos Hôpitaux, porteurs d'une technicité en prothèse de hanche que certains plus anciens n'accueillaient qu'avec précaution; ils avaient tellement enlevé de prothèses acryliques mal supportées.

Mais il apparaissait alors à tous que le patchwork qui unissait la Traumatologie aiguë, l'Orthopédie froide et la Chirurgie Plastique, se trouvait devant une évolution inévitable.

La Chirurgie Plastique et Reconstructive s'isolait et divorçait d'un mariage avec des activités qui n'étaient pas les leurs.

L'Orthopédie et la Traumatologie avaient souvent du mal à trouver leurs marques.

Il était parfois difficile à faire cohabiter une Orthopédie faite toute de programmation, de calme et d'hyper-asepsie, avec une traumatologie qui se devait à l'urgence qui nécessite disponibilité, souplesse et l'implication d'autres spécialités viscérales ou neurologiques.

Les traumatismes se rient des fron- 
tières anatomiques et des limites des spécialités : Neuro-Chirurgiens, Chirurgiens Maxillo-faciaux, Chirurgiens thoraciques, Chirurgiens abdominaux et vasculaires, avaient leur rôle à jouer et revendiquaient à juste titre une place en Traumatologie car elle comportait les gestes de leur spécialité et leur apportait un recrutement nécessaire, mais ils la redoutaient aussi par le désordre qu'apporte l'urgence dans un Service pas toujours adapté.

Je ne suis pas sûr que des solutions aient été trouvées partout dans notre pays mais je me réjouis que dans l'Est de la France l'organisation soit souvent satisfaisante.

La disparition de la Chirurgie Générale ne peut pas ne pas poser des problèmes. Je suis probablement un des derniers Chirurgiens à avoir pu ou dû réaliser toute la palette des inter- ventions chirurgicales. Cette pratique est évidemment périmée, mais inversement, l'hyper-spécialisation qui a été l'ambition des jeunes et le refuge des Anciens, a presque tari la source des Chirurgiens susceptibles de faire face à la Chirurgie en situation de catastrophe sur laquelle insistaient dans un Colloque récent les Chirurgiens de l'Ecole du PHARO à Marseille.

Il faut que nos jeunes confrères conservent une certaine compétence dans les spécialités voisines pour ne pas risquer un jour de se trouver désarmés devant des problèmes de polytraumatologie pour lesquels le découpage de la Chirurgie entre Spécialistes multiples empêcherait de trouver les solutions efficaces et simples pour un Maître d'oeuvre unique.

Ces quelques lignes ne sont pas une lamentation désabusée, mais au contraire, un chant de joie et d'espoir devant l'évolution du merveilleux métier qui est le nôtre.

Il faudrait parler aussi de l'évolution des Sociétés Scientifiques. Dans l'Est, elle est particulièrement féconde. J'ai été très fier et très heureux d'être membre du GECO et de la S.O.T.EST dont j'ai été le Premier Président de la région Bourgogne.

La revue "Orthopédie-Traumatologie", dont la vocation est européenne à l'aube de 1993, est le symbole de la vitalité de l'Orthopédie et de la Traumatologie, deux soeurs jumelles aussi séduisantes l'une que l'autre.

Merci à sa rédaction de m'avoir permis de m'y exprimer, et je souhaite que nos jeunes Collègues aient la joie de vivre la même évolution de notre métier que celle que leurs anciens ont eu la chance de connaître. 